\title{
ATTRACTING DOMAINS FOR SEMI-ATTRACTIVE TRANSFORMATIONS OF $\mathbf{C}^{p}$
}

\author{
MONIQUE HAKIM
}

\begin{abstract}
Let $F$ be a germ of analytic transformation of $\left(\mathbf{C}^{p}, 0\right)$. We say that $F$ is semi-attractive at the origin, if $F_{(0)}^{\prime}$ has one eigenvalue equal to 1 and if the other ones are of modulus strictly less than 1. The main result is: either there exists a curve of fixed points, or $F$ - Id has multiplicity $k$ and there exists a domain of attraction with $k-1$ petals. We study also the case where $F$ is a global isomorphism of $\mathbf{C}^{2}$ and $F$ - Id has multiplicity $k$ at the origin. This work has been inspired by two papers: one of P. Fatou (1924) and the other one of T. Ueda (1986).
\end{abstract}

\section{Introduction}

Let $F$ be a germ of analytic transformation from $\left(\mathbf{C}^{p}, 0\right)$ to $\left(\mathbf{C}^{p}, 0\right)$, i.e., a holomorphic map defined in a neighborhood of the origin in $\mathbf{C}^{p}$ which leaves the origin $0=(0,0)$ of $\mathbf{C}^{p}$ fixed. We are interested in the behavior of the iterates $\left(x_{n}, y_{n}\right)=F^{(n)}(x, y)$ of points $(x, y)$ near the origin. We implicitly assume that they are defined. We study the situation where $F_{(0)}^{\prime}$ has one eigenvalue equal to 1 and the others are $\left\{\lambda_{j}\right\}_{2 \leq j \leq p}$, with $0 \leq\left|\lambda_{j}\right|<1$. We call semi-attractive such trasnformations. We want to investigate the existence of attracting domains at 0 in a neighbourhood of 0 . As the partial derivative $\frac{\partial}{\partial x_{1}} F_{1}^{(n)}(0)=1$ in some coordinate system, the family $\left\{F^{(n)}\right\}$ cannot converge to 0 in a neighborhood of 0 . So by attracting domains in a neighborhood of 0 , we mean open domains $D$ with $0 \in \partial D$ such that $x_{n}=F^{(n)}(x)$ converge to 0 for $x \in D$.

When $p=1$, the dynamics of analytic transformations from $(\mathbf{C}, 0)$ to $(\mathbf{C}, 0)$ with eigenvalue 1 , i.e., transformations which can be written with convergent power series in $x$ as

$$
F(x)=x_{1}=x\left(1+a_{1} x+a_{2} x^{2}+\ldots\right)
$$


have been studied by Fatou and Leau. Their theory is quite complete (see for instance $[\mathbf{B}]$ ).

In his paper $[\mathbf{F}]$ on transformations of $\left(\mathbf{C}^{2}, 0\right)$ Fatou investigates the case of transformations with eigenvalues 1 and $b$, with $0<|b|<1$. He proves the existence of a coordinate system $(x, y)$ where $F$ can be written

$$
\left\{\begin{array}{l}
x_{1}=f(x, y)=a_{1}(y) x+a_{2}(y) x^{2}+\ldots \\
y_{1}=g(x, y)=b y+b_{1}(y) x+b_{2}(y) x^{2}+\ldots
\end{array}\right.
$$

where the $a_{j}(y), b_{j}(y)$ are holomorphic functions in a neighborhood of $0 \in \mathbf{C}$ such that $a_{1}(0)=1, b_{1}(0)=0$, the $x$-coordinate being chosen in such a way that $\{x=0\}$ is the invariant curve of Poincaré. Then Fatou shows that, if $a_{2}(0) \neq 0$, there exists an attracting domain at 0 . The projection on the $x$-plane of the dynamics is of the same type as the one we get in $\mathbf{C}$ with the $a_{j}$ 's constant. This case has been studied again by Ueda $[\mathbf{U}]$ with a better reduced form for $F$, which allows him to give a simple and more complete description of the domain of convergence.

When $y=0$ is a curve of fixed points, the transformation $F$ is of type

$$
\left\{\begin{array}{l}
x_{1}=x+x y f(x, y) \\
y_{1}=y\left(b+b_{1}(y) x+b_{2}(y) x^{2}+\ldots\right)
\end{array},\right.
$$

a case considered by Lattès $[\mathbf{L}]$. Fatou shows that the coordinates can be chosen in such a way that we get the reduced form

$$
\left\{\begin{array}{l}
x_{1}=x \\
y_{1}=y\left(b+\sum_{i+j \geq 1} b_{i j} x^{i} y^{j}\right) .
\end{array}\right.
$$

So a neighborhood of 0 is attracted by the curve of fixed points along the trajectories $x=$ constant. Then Fatou asks if there exis other cases for which there is no attracting domain at 0 . He asks what happens for instance with a transformation like

$$
\left\{\begin{array}{l}
x_{1}=\frac{x}{1+x y} \\
y_{1}=b y+x^{2}
\end{array} ?\right.
$$

In this paper, we will see that there is an attracting domain of 0 . We prove the following result. 
Theorem 1.1. Let $F$ be an analytic germ of transformation from $\left(\mathbf{C}^{p}, 0\right)$ to $\left(\mathbf{C}^{p}, 0\right)$, with eigenvalues in $0\left\{1,\left\{\lambda_{j}\right\}_{2 \leq j \leq p}\right\}$, such that $0 \leq$ $\left|\lambda_{j}\right|<1$, for $2 \leq j \leq p$, then either there exists a curve of fixed points or there exists an attracting domain of 0 .

More precisely, let Id be the identity of $\left(\mathbf{C}^{p}, 0\right)$. Either there exists a curve of fixed points or $F$ - Id has a finite multiplicity $k \geq 2$. We show that in the case of multiplicity $k \geq 2$, there exists an attracting domain $D$ of 0 made of $k-1$ attracting petals, i.e. $k-1$ disjoint open sets $\left\{D_{j}\right\}$ positively invariant by $F$ such that $0 \in \partial D_{j}$ and that every $x \in D_{j}$ is attracted by 0 . Conversely, if a point $x$ is attracted by 0 , for $n$ big enough, $x_{n}=F^{(n)}(x)$ is in one of the $D_{j}$.

Let us recall (see for instance $[\mathbf{C}]$ ) the definition of the multiplicity of a holomorphic transformation $\Phi$ from $\left(\mathbf{C}^{p}, 0\right)$ to $\left(\mathbf{C}^{p}, 0\right)$ such that 0 is isolated in the fiber $\Phi^{-1}(\{0\})$. Let $V$ be a compact neighborhood of 0 such that the restriction $\Phi_{V}$ of $\Phi$ to $V$ is proper from $V$ to $W=\Phi(V)$; let $\operatorname{Br}(\Phi)$ be the branched locus of $\Phi$, i.e. the set of $z \in V$ where $\operatorname{Det}(J(\Phi)(z))=0$; here $J(\Phi)$ is the matrix $\left(\frac{\partial \Phi_{i}}{\partial z_{j}}\right)_{1 \leq i \leq p, 1 \leq j \leq p}$. The multiplicity of $\Phi$ at 0 is then the number of points in a fiber $\Phi^{-1}(\{\zeta\})$ for $\zeta$ a point in $W$ which does not belong to $\Phi(\operatorname{Br}(\Phi))$. We use then the lemma

Lemma 1.2 [C, p. 102]. Let $\Phi$ be a holomorphic transformation $\Phi$ from $\left(\mathbf{C}^{p}, 0\right)$ to $\left(\mathbf{C}^{p}, 0\right)$ such that 0 is isolated in its fiber $\Phi^{-1}(\{0\})$. Suppose that the matrix $\left(\frac{\partial \Phi_{i}}{\partial z_{j}}\right)_{2 \leq i \leq p, 2 \leq j \leq p}$ has rank $p-1$. Let $C$ be the curve $\left\{z_{j}=\varphi_{j}\left(z_{1}\right)\right\}_{2 \leq j \leq p}$ defined via the implicit function theorem by $\Phi_{2}=\Phi_{3}=\cdots=\Phi_{p}=0$, then the multiplicity of $\Phi$ at 0 is equal to the multiplicity at 0 of the function of one variable

$$
\Phi_{1}\left(z_{1}, \varphi_{2}\left(z_{1}\right), \ldots, \varphi_{p}\left(z_{1}\right)\right) .
$$

Proof of Lemma 1.2: From the relations

$$
\frac{\partial \Phi_{i}}{\partial z_{1}}+\sum_{j=2}^{p} \varphi_{j}^{\prime}\left(z_{1}\right) \frac{\partial \Phi_{i}}{\partial z_{j}}=0, \text { for } 2 \leq i \leq p
$$

on $C$, we get

$$
\operatorname{Det}\left(J(\Phi)_{\mid C}\right)=\left(\frac{\partial \Phi_{1}}{\partial z_{1}}+\sum_{j=2}^{p} \varphi_{j}^{\prime}\left(z_{1}\right) \frac{\partial \Phi_{1}}{\partial z_{j}}\right) \operatorname{Det}\left(\frac{\partial \Phi_{i}}{\partial z_{j}}\right)_{2 \leq i \leq p, 2 \leq j \leq p} .
$$


As 0 is isolated in its fiber $\Phi^{-1}(\{0\})$,

$$
d \Phi_{1 \mid C}=\frac{\partial \Phi_{1}}{\partial z_{1}}+\sum_{j=2}^{p} \varphi_{j}^{\prime}\left(z_{1}\right) \frac{\partial \Phi_{1}}{\partial z_{j}},
$$

is not identically zero. Hence $C$ is not in $\operatorname{Br}(\Phi)$. So to count the multiplicity, we can restrict $\zeta$ to $\Phi(C)$, and we have just to count the zeros of $\phi_{1}\left(z_{1}\right)=\Phi_{1}\left(z_{1}, \varphi_{2}\left(z_{1}\right), \ldots, \varphi_{p}\left(z_{1}\right)\right)=\zeta_{1}$ for $\zeta_{1}$ in a neighborhood of 0 in C. By Rouché's theorem, this multiplicity is given by the order in 0 of $\phi_{1}$.

Let us for instance, compute the multiplicity of $\phi=F$ - Id in the example (1.3). According to lemma 1.2, we solve the equation

$$
y=b y+x^{2}
$$

and replace $y$ in the first relation. We get

$$
x_{1}=\frac{x}{1+\frac{x^{3}}{1-b}}=x\left(1-\frac{x^{3}}{1-b}+\ldots\right) .
$$

The multiplicity of $F-$ Id at 0 is 4 . By theorem 1 we see that there exists an attracting domain at 0 with three petals.

The result is also true if $F_{(0)}^{\prime}$ is not invertible. For instance the transformation

$$
\left\{\begin{array}{l}
x_{1}=x\left(1+x y-x^{3}\right) \\
y_{1}=y^{2}+x^{2}
\end{array}\right.
$$

where $F_{(0)}^{\prime}$ has for eigenvalues 1 et $0, F$ - Id has multiplicity 6 in 0. Indeed, solve

$$
y=y^{2}+x^{2},
$$

we get

$$
y=x^{2}+x^{4}+\ldots,
$$

replacing $y$ in the first relation

$$
x_{1}-x=x^{2} y-x^{4}=x^{6}+o\left(x^{6}\right) .
$$

So there is an attracting domain at 0 with 5 petals.

In $[\mathbf{U}]$, Ueda studies the analytic transformations of $\left(\mathbf{C}^{2}, 0\right)$ with eigenvalues $\{1, b\}$ at 0 , such that $0<|b|<1$. He calls them transformations of type $(1, b)$. Then he defines a classification $\left\{(1, b)_{k}\right\}$, for $k$ integer, 
$1 \leq k \leq+\infty$, on these transformations. In fact, the integer $k+1$ for type $(1, b)_{k}$ of Ueda is precisely the multiplicity of $F-$ Id. Ueda concentrates his work on the case $(1, b)_{1}$. This is the case considered by Fatou when in the expression (1.1), we have $a_{2}(0) \neq 0$. Ueda introduces transformations of the coordinates which give simple reduced forms to study the attracting domain, in the case $a_{2}(0) \neq 0$. Similar transformations will be used here.

Ueda studies also the case of a global automorphism $F$ in $\mathbf{C}^{2}$. Let $F$ be a global automorphism in $\mathbf{C}^{2}$ with a fixed point of type $(1, b)_{1}$, he proves that, like in the examples given by Fatou and Bieberbach with eigenvalues of modulus strictly less than 1, the attracting domain is isomorphic to $\mathbf{C}^{2}$. This is, for instance, the case for the attraction domain of the Hénon transformation

$$
\left\{\begin{array}{l}
x_{1}=x(1+b)-b y+x^{2}, \\
y_{1}=x
\end{array},\right.
$$

for $0<|b|<1$. This statement has the following generalization.

Theorem 1.3. Let $F$ be a global automorphism in $\mathbf{C}^{2}$ with a fixed point $p$, such that $F_{(p)}^{\prime}$ has eigenvalues $\{1, \lambda\}$, with $|\lambda|<1$, and that $F-I d$ has a multiplicity $k+1$ in $p$. The attracting domain of $p$ has then $k$ components and each component is isomorphic to $\mathbf{C}^{2}$.

Theorem 1.3 applies for instance to Hénon transformations

$$
\left\{\begin{array}{l}
x_{1}=x(1+b)-b y+P(x) \\
y_{1}=x
\end{array}\right.
$$

where $P$ is a polynomial with a zero of order $k+1$ at the origin.

\section{Reduced forms of semi-attractive transformations}

Proposition 2.1. Let $F$ be a semi-attractive germ of transformation of $\left(\mathbf{C}^{p}, 0\right)$, with eigenvalues $\left\{1,\left\{\lambda_{j}\right\}_{2 \leq j \leq p}\right\}, 0 \leq\left|\lambda_{j}\right|<1$, for $2 \leq j \leq p$. There exist coordinates $(x, y), x \in \mathbf{C}, y \in \mathbf{C}^{p-1}$ in which $F$ has the form

$$
\left\{\begin{array}{l}
x_{1}=u(x, y)=a_{1}(y) x+a_{2}(y) x^{2}+\ldots \\
y_{1}=v(x, y)=g(y)+x h(x, y)
\end{array},\right.
$$

where $\left\{a_{j}().\right\}, j=1,2, \ldots, g($.$) and h(.,$.$) are respectively germs of holo-$ morphic functions from $\left(\mathbf{C}^{p-1}, O\right)$ to $\mathbf{C}$, from $\left(\mathbf{C}^{p-1}, O\right)$ to $\mathbf{C}^{p-1}$ and 
from $\left(\mathbf{C}^{p}, O\right)$ to $\mathbf{C}^{p-1}$, with $a_{1}(0)=1, g(0)=0, h(0,0)=0$, and $g_{(0)}^{\prime}$ is triangular with eigenvalues $\left\{\lambda_{j}\right\}_{2 \leq j \leq p}$.

Proof: Let $E_{1} \oplus E_{2}$ be the Jordan decomposition of $\mathbf{C}^{p}$ in characteristic subspaces. Here $E_{1}$ is associated to the eigenvalue 1 and $E_{2}$ to the set of the eigenvalues $\left\{\lambda_{j}\right\}_{2 \leq j \leq p}$. There exists an analytic stable submanifold $X$ attracted by 0 and tangent to $E_{2}$ (see $[\mathbf{R}]$ for a sketch of the proof and a complete bibliography). We then just choose coordinates $(x, y)$, $x \in \mathbf{C}, y \in \mathbf{C}^{p-1}$, in such a way that $X$ is $\{x=0\}$ and that matrix $F_{(0)}^{\prime}$ is triangular.

Proposition 2.2. Let $F$ be a semi-attractive germ of transformation of $\left(\mathbf{C}^{p}, 0\right)$. For every integer $m$ there exists coordinates $(x, y), x \in \mathbf{C}$, $y \in \mathbf{C}^{p-1}$, in which the transformation has the form

$$
\left\{\begin{array}{l}
x_{1}=x+a_{2} x^{2}+\ldots a_{m} x^{m}+a_{m+1}(y) x^{m+1}+\ldots \\
y_{1}=g(y)+x h(x, y)
\end{array},\right.
$$

i.e. like in (2.1), but with $a_{1}=1$ and $a_{2}, \ldots, a_{m}$ are constants.

Remark. This proposition is in $[\mathbf{U}]$ in the case of a semi-attractive invertible germ of $\left(\mathbf{C}^{2}, 0\right)$. The following proof is just a generalization of it.

Proof: We start with

$$
\left\{\begin{array}{l}
x_{1}=a_{1}(y) x+a_{2}(y) x^{2}+\ldots \\
y_{1}=g(y)+x h(x, y)
\end{array}\right.
$$

and we proceed inductively on $h$.

1) Reduction to $a_{1}(y)=1$. We use the coordinate transformation

$$
\left\{\begin{array} { l } 
{ X = u ( y ) x } \\
{ Y = y }
\end{array} \text { or } \quad \left\{\begin{array}{l}
s=X / u(Y) \\
y=Y
\end{array}\right.\right.
$$

with $u($.$) a germ of analytic function from \left(\mathbf{C}^{p-1}, 0\right)$ to $\mathbf{C}$ such that $u(0)=1$, to be chosen. We want

$$
\begin{aligned}
X_{1} & =u\left(y_{1}\right) x_{1}=u(g(y)+x h(x, y))\left[a_{1}(y) x+a_{2}(y) x^{2}+\ldots\right] \\
& =u(g(Y)+\ldots)\left[a_{1}(Y) X / u(Y)+\ldots\right] \\
& =\frac{a_{1}(Y) u(g(Y))}{u(Y)} X+O\left(X^{2}\right)=X+O\left(X^{2}\right) .
\end{aligned}
$$


So we must choose $u$ such that

$$
\begin{aligned}
& u(Y)=a_{1}(Y) u(g(Y)) \\
& u(g(Y))=a_{1}\left(g(Y) u\left(g^{(2)}(Y)\right)\right. \\
& \ldots \\
& u\left(g^{(n)}(Y)\right)=a_{1}\left(g^{(n)}(Y)\right) u\left(g^{(n+1)}(Y)\right) .
\end{aligned}
$$

This gives for $u$ the unique solution

$$
u(Y)=\prod_{n=0}^{\infty} a_{1}\left(g^{(n)}(Y)\right) .
$$

Since $a_{1}(0)=1$ and since there exists $\alpha, 0<\alpha<1$, such that for $\|y\|$ small enough, one has $\|g(y)\| \leq \alpha\|y\|$, so $\left\|g^{(n)}(y)\right\| \leq \alpha^{n}\|y\|$, the infinity product above is convergent in a neighborhood of 0 .

2) Suppose that for $m \geq 2$, with some coordinates $(x, y), F$ takes the form

$$
\left\{\begin{array}{l}
x_{1}=x+a_{2} x^{2}+\ldots a_{m-1} x^{m-1}+a_{m}(y) x^{m}+\ldots \\
y_{1}=g(y)+x h(x, y)
\end{array},\right.
$$

with the $a_{j}$ 's constant for $1 \leq j \leq m-1$. We then use a coordinate transformation

$$
\left\{\begin{array} { l } 
{ X = x + v ( y ) x ^ { m } } \\
{ Y = y }
\end{array} \text { or } \left\{\begin{array}{l}
x=X-v(Y) X^{m}+\ldots \\
y=Y
\end{array},\right.\right.
$$

with $v(y)$ a holomorphic function in a neighborhood of 0 in $\mathbf{C}^{p-1}$ such that $v(0)=0, v$ to be chosen. We get

$$
\begin{aligned}
X_{1} & =x_{1}+v\left(y_{1}\right) x_{1}^{m} \\
& =x+a_{2} x^{2}+\ldots a_{m-1} x^{m-1}+a_{m}(y) x^{m}+v(g(y)) x^{m}+O\left(x^{m+1}\right) \\
& =X-v(Y) X^{m}+a_{2} X^{2}+\ldots a_{m-1} X^{m-1}+a_{m}(y) X^{m} \\
& +v(g(y)) X^{m}+O\left(X^{m+1}\right) .
\end{aligned}
$$

So we need that

$$
\begin{aligned}
& v(Y)-v(g(Y))=a_{m}(y)-a_{m}(0) \\
& v(g(Y))-v\left(g^{2}(Y)\right)=a_{m}(g(y))-a_{m}(0) \\
& \cdots \\
& v\left(g^{n}(Y)\right)-v\left(g^{n+1}(Y)\right)=a_{m}\left(g^{n}(y)\right)-a_{m}(0) .
\end{aligned}
$$

The unique solution is then

$$
v(y)=\sum_{n=0}^{\infty}\left\{a_{m}\left(g^{n}(y)\right)-a_{m}(0)\right\} .
$$

The series converges in a neighborhood of 0 because $g$ is contraction and because $a_{m}(y)-a_{m}(0)=0$ for $y=0$. 
Proposition 2.3. Let $F$ be a semi-attractive germ of transformation of $\left(\mathbf{C}^{p}, 0\right)$, such that $F$ - Id is of multiplicity $k+1$ in 0. Then the transformation can be written in some coordinates $(x, y), x \in \mathbf{C}, y \in$ $\mathbf{C}^{p-1}$

$$
\left\{\begin{array}{l}
x_{1}=x\left(1+x^{k}+C x^{2 k}+a_{2 k+1}(y) x^{2 k+1}+\ldots\right) \\
y_{1}=g(y)+x h(x, y)
\end{array},\right.
$$

with $C$ a constant.

Proof: Assume then that the transformation is written in the form (2.2) with $m>k+1$. We want to evaluate the multiplicity of $F$ - Id at 0 . Since $\left(I_{p-1}-g^{\prime}\right)(0)$ is invertible, we can use lemma 1.2. Using the implicit function theorem, we can solve locally in $y=y(x)$ the equation $y=y_{1}$. The multiplicity is then given by the order at the origin of

$$
x_{1}-x=a_{2} x^{2}+\ldots a_{m} x^{m}+a_{m+1}(y) x^{m+1}+\ldots .
$$

Since $F-$ Id is of multiplicity $k+1$ in 0 , we have $a_{1}=\cdots=a_{k}=0$ and $a_{k+1} \neq 0$. If we use proposition (2.2) with $m=2 k+1$, we get

$$
x_{1}=x\left(1+a_{k+1} x^{k}+\cdots+a_{2 k+1} x^{2 k}+a_{2 k+2}(y) x^{2 k+1}+\ldots\right) .
$$

As in the case of one variable, a polynomial transformation in the single variable $x$ leads to the required form. The coefficient of $x^{k+1}$ can be an arbitrary constant not equal to 0 , the coefficient of $x^{2 k+1}$ is then fixed (see for instance [B, theorem 6.5.7, page 122].

\section{Existence of attracting domains and curve of fixed points}

We will now prove the theorem 1.1 stated in the introduction. Let $F$ be a semi-attractive germ of transformation of $\left(\mathbf{C}^{p}, 0\right)$, given as before in the form

$$
\left\{\begin{array}{l}
x_{1}=u(x, y)=x\left(1+a_{2}(y) x+\ldots\right) \\
y_{1}=v(x, y)=g(y)+x h(x, y)
\end{array},\right.
$$

with $x \in \mathbf{C}, y \in \mathbf{C}^{p-1}$ and $g, h$ like in proposition (2.1). To find the fixed points of $F$, one can first solve locally in $y=y(x)$ the relation $y=v(x, y)$, thus obtaining an analytic curve $y=\varphi(x)$. There exists a curve of fixed points if and only if the relation

$$
x=u(x, \varphi(x))
$$

is also satisfied. If not, $F$-Id is of finite multiplicity, and the multiplicity is given by the order at 0 of $x-u(x, \varphi(x))$.

The following corollary is an answer to a question of Fatou [F, page 131]) who asked if for such $F$, there could exist a curve of fixed points through 0 for some iterate $F^{(n)}$ but not for $F$. 
Corollary. Let $F$ be a semi-attractive germ of transformation of $\left(\mathbf{C}^{p}, 0\right)$. There exists a curve of fixed points through 0 for an iterate $F^{(n)}$ of $F$ if and only if there is one for $F$.

Proof: It is an immediate consequence of proposition (2.3), for if $F$ is in the form (2.3), we have

$$
x_{n}=x\left(1+n x^{k}+\ldots\right),
$$

so $F^{(n)}$ - Id has the same multiplicity as $F$ - Id.

The proof of theorem 1.1 is then a consequence of the following proposition.

Proposition 3.1. Let $F$ be a semi-attractive germ of transformation of $\left(\mathbf{C}^{p}, 0\right)$, of multiplicity $k+1$ in 0 , then there exists an attracting domain with $k$ petals.

Proof: We can suppose that $F$ is in the form given by proposition (2.3)

$$
\left\{\begin{array}{l}
x_{1}=x\left(1+a_{k} x^{k}+a_{2 k} x^{2 k}+a_{2 k+1}(y) x^{2 k+1}+\ldots\right) \\
y_{1}=g(y)+x h(x, y)
\end{array},\right.
$$

with $a_{k} \neq 0$. Changing $x$ in some $\lambda x$ one can assume $a_{k}=-\frac{1}{k}$. We can then imitate a Fatou's method simplified here by using the reduced form for $F$ which gives easily the Abel-Fatou invariant functions.

Let $R$ and $\rho$ be positive constants to be adjusted later. The half complex-plane $P_{R}$ and the subset $V_{R, \rho}$ of $\mathbf{C} \times \mathbf{C}^{p-1}$ are defined by

$$
\begin{aligned}
P_{R} & =\{X \in \mathbf{C} ; \operatorname{Re} X \geq R\}, \\
V_{R, \rho} & =\left\{(X, y) \in \mathbf{C} \times \mathbf{C}^{p-1} ; X \in P_{R},\|y\|<\rho\right\} .
\end{aligned}
$$

Let $D_{R}$ and $U_{R, \rho}$ be the images of $P_{R}$ and $V_{R, \rho}$ by the inversion $z=\frac{1}{X}$, so we have

$$
\begin{aligned}
D_{R} & =\left\{z \in \mathbf{C} ;\left|z-\frac{1}{2 R}\right|<\frac{1}{2 R}\right\}, \\
U_{R, \rho} & =\left\{(z, y) \in \mathbf{C} \times \mathbf{C}^{p-1} ; z \in D_{R},\|y\|<\rho\right\} .
\end{aligned}
$$

There are $k$ branches of $z^{1 / k}$ in $D_{R}$. Let $\left\{\Delta_{R_{j}}\right\}_{0 \leq j \leq k-1}$ be the images of $D_{R}$ by these determinations. We will show that, for $R$ big enough and $\rho$ small enough, the domains

$$
W_{R, \rho, j}=\left\{(x, y) \in \mathbf{C} \times \mathbf{C}^{p-1} ; x \in \Delta_{R j},\|y\|<\rho\right\}, \quad 0 \leq j \leq k-1,
$$


are attracting domains.

Raising the relation

$$
x_{1}=x\left(1-\frac{1}{k} x^{k}+a_{2 k} x^{2 k}+\ldots\right)
$$

to the power $k$, we get

$$
\begin{aligned}
x_{1}^{k} & =x^{k}\left(1-\frac{1}{k} x^{k}+a_{2 k} x^{2 k}+\ldots\right)^{k} \\
& =x^{k}\left(1-x^{k}+c_{2 k} x^{2 k}+c_{2 k+1}(y) x^{2 k+1}+\ldots\right) \\
y_{1} & =g(y)+x h(x, y) .
\end{aligned}
$$

We then restrict $(x, y)$ to a $W_{R, \rho, j}$ for fixed $R, \rho, j$, and we make the transformations

$$
\left(z=x^{k}, y=1\right) \text { from } W_{R, \rho, j} \text { to } U_{R, \rho},
$$

and

$$
\left(X=\frac{1}{z}, y=y\right) \text { from } U_{R, \rho} \text { to } V_{R, \rho} .
$$

For $R$ big enough and $\rho$ small enough, the transformation $F$ is defined in $V_{R, \rho}$, where we get

$$
\begin{aligned}
X_{1} & =\frac{X}{1-x^{k}+c_{2 k} x^{2 k}+c_{2 k+1}(y) x^{2 k+1}+\ldots} \\
& =X\left(1+\frac{1}{X}+c \frac{1}{X^{2}}+\alpha(y) \frac{x}{X^{2}}+\ldots\right) .
\end{aligned}
$$

So $F$ becomes

$$
\left\{\begin{array}{l}
X_{1}=X+1+c \frac{1}{X}+O_{y}\left(\frac{1}{|X|^{1+1 / k}}\right) \\
y_{1}=g(y)+x h(x, y)=g(y)+O_{y}\left(\frac{1}{|X|^{1 / k}}\right)
\end{array} .\right.
$$

Here the notation $O_{y}\left(\frac{1}{|X|^{\alpha}}\right)$ represents a holomorphic function of $(X, y)$ in $V_{R, \rho}$ which is bounded by $\frac{K}{|X|^{\alpha}}$ for some constant $K$.

Let $K$ be a constant such that

$$
\left\{\begin{array}{l}
\left|X_{1}-X-1\right| \leq \frac{K}{|X|} \leq \frac{K}{R} \\
\left\|y_{1}-g(y)\right\| \leq \frac{K}{|X|^{1 / k}} \leq \frac{K}{R^{1 / k}}
\end{array},\right.
$$


in $V_{R, \rho}$. Since $g$ is a contraction, there exists $b, 0<b<1$, such that for $\rho$ small enough we get in $\|y\| \leq \rho$

$$
\|g(y)\| \leq b\|y\| .
$$

The condition $\frac{K}{R}<\frac{1}{2}$ implies $\operatorname{Re} X_{1} \geq \operatorname{Re} X+\frac{1}{2}$ and the condition

$$
\frac{K}{R^{1 / k}}<(1-b) \rho
$$

implies $\left\|y_{1}\right\| \leq\|y\| \leq \rho$. So for $R$ big enough, $V_{R, \rho}$ is mapped to itself.

Then to prove that $W_{R, \rho, j}$ is attracted by 0 , we have to show that $V_{R, \rho}$ is attracted by $(\infty, 0)$. We see inductively that

$$
\operatorname{Re} X_{n} \geq R+\frac{n}{2}
$$

Let $C$ be a constant big enough to have $C \geq \frac{2 K}{1-b}$, and $\rho \leq \frac{C}{(R)^{1 / k}}$, we prove by induction that, if $R$ is big enough, we have

$$
\left\|y_{n}\right\| \leq \frac{C}{\left(R+\frac{n}{2}\right)^{1 / k}} .
$$

Indeed, since

$$
\left\|y_{n+1}\right\| \leq b\left\|y_{n}\right\|+\frac{K}{\left|X_{n}\right|^{1 / k}} \leq \frac{b C+K}{\left(R+\frac{n}{2}\right)^{1 / k}} .
$$

The inequality

$$
\left\|y_{n+1}\right\| \leq \frac{C}{\left(R+\frac{n+1}{2}\right)^{1 / k}}
$$

will be satisfied if we have

$$
\left(\frac{R+\frac{1}{2}}{R}\right)^{1 / k} \leq \frac{C}{b C+K} .
$$

But from $C \geq \frac{2 K}{1-b}$, we see that $\frac{C}{b C+K} \geq \frac{2}{1+b}>1$. So that (3.5) is true if $R$ is big enough.

We have now $k$ disjoint domains attracted by 0 . Each of them is positively invariant by $F$ since $V_{R, \rho}$ is positively invariant, and, since $x_{n+1} \sim x_{n}$ when $n \rightarrow \infty$, we have always the same branch of $x^{1 / k}$. Let $D$ be the attracting domain of 0 , we want to prove that if $\zeta \in D$, for $n$ 
big enough, $\zeta_{n}=\left(x_{n}, y_{n}\right)$ is in one of the $W_{R, \rho, j}$ 's, or equivalently that $\left(x_{n}^{k}, y_{n}\right)$ is in $U_{R, \rho}$, or that $\left(\frac{1}{x_{n}^{k}}, y_{n}\right)$ is in $V_{R, \rho}$. But $y_{n} \rightarrow 0$ and we have

$$
\frac{1}{x_{1}^{k}}=\frac{1}{x^{k}}+1+c x^{k}+O_{y}\left(x^{k+1}\right)
$$

so $\operatorname{Re} \frac{1}{x_{n}^{k}} \rightarrow \infty$ when $x_{n} \rightarrow 0$. So $\zeta$ belongs to the union of the increasing sequence of open sets

$$
D_{j}=\bigcup_{n=0}^{\infty} F^{-n}\left(W_{R, \rho, j}\right) .
$$

When $F$ is an isomorphism of $\mathbf{C}^{p}$, each $D_{j}$ is connected. In general, we can only say that the $D_{j}$ 's are disjoint and contain the $W_{R, \rho, j}$ 's.

\section{Abel-Fatou's functions}

Let $F$ be a semi-attractive germ of $\left(\mathbf{C}^{p}, 0\right)$ such that $F$ - Id has multiplicity $k+1$ in 0 , the coordinates and the notations used here are those introduced in the proof of proposition 3.1. In each attractive petal $W_{R, \rho, j}$, we can define an Abel-Fatou function $\varphi$, more precisely, a holomorphic function $\varphi: W_{R, \rho, j} \rightarrow \mathbf{C}$ verifying the functional equation

$$
\varphi(F(p))=\varphi(p)+1,
$$

in the following way.

To construct $\varphi$ we first observe that in $V_{R, \rho}=\left\{(X, y) \in \mathbf{C} \times \mathbf{C}^{p-1}\right.$; $\operatorname{Re} X \geq R,\|y\|<\rho\}, F$ is given (see (3.3)) by

$$
\left\{\begin{array}{l}
X_{1}=X+1+c \frac{1}{X}+O_{y}\left(\frac{1}{|X|^{1+1 / k}}\right) \\
y_{1}=g(y)+x h(x, y)=g(y)+O_{y}\left(\frac{1}{|X|^{1 / k}}\right)
\end{array} .\right.
$$

Following Fatou, define

$$
U_{n}=X_{n}-n-c \log X_{n} .
$$

We get

$$
\left\|U_{n+1}-U_{n}\right\|=\left\|X_{n+1}-X_{n}-1-c \log \frac{X_{n+1}}{X_{n}}\right\| \leq \frac{K}{n^{1+1 / k}} .
$$


So the series $\sum_{n=0}^{\infty}\left(U_{n+1}-U_{n}\right)$ is uniformly convergent and has a holomorphic bounded sum in $V_{R, \rho}$. Hence

$$
U_{n}(X, y)=U_{0}+\sum_{k=0}^{n-1}\left(U_{k+1}-U_{k}\right)
$$

has a limit

$$
u(X, y)=X-c \log X+v(x, y)
$$

with $v($,$) a holomorphic bounded function. The functional equation$

$$
u\left(X_{1}, y_{1}\right)=u(X, y)+1
$$

is an immediate consequence of

$$
u\left(X_{1}, y_{1}\right)=\lim _{n \rightarrow \infty}\left(X_{n+1}-n-c \log X_{n+1}\right) .
$$

In each attractive petal $W_{R, \rho, j}$, the function $\varphi: W_{R, \rho, j} \rightarrow \mathbf{C}$ is then defined by

$$
\varphi(x, y)=u\left(\frac{1}{x^{k}}, y\right)
$$

and verifies $\varphi(f(p))=\varphi(p)+1$. Remark that in $W_{R, \rho, j}$, the function $\varphi$ has the asymptotic expansion

$$
\begin{aligned}
\varphi(x, y) & =\frac{1}{x^{k}}\left(1-c x^{k} \log x^{k}+x^{k} v_{1}\left(x^{k}, y\right)\right) \\
& =\frac{1}{x^{k}}\left(1+O\left(\left|x^{k} \log x^{k}\right|\right)\right) .
\end{aligned}
$$

\section{Global isomorphism of $\mathrm{C}^{2}$}

We now consider the case where $F$ is an isomorphism of $\mathbf{C}^{2}$, with fixed point 0 . We assume that $F_{(0)}^{\prime}$ is semi-attractive (with eigenvalues 1 and $\lambda$ s.t. $|\lambda|<1)$ and that $F-$ Id has multiplicity $k+1$ in 0 . In this case the attracting domain has $k$ components given with the notations of proposition (3.1) by

$$
D_{j}=\bigcup_{n=0}^{\infty} F^{-n}\left(W_{R, \rho, j}\right)
$$

for $j=0,1, \ldots, k-1$. For a fixed $j$, the Abel-Fatou's function defined in $W_{R, \rho, j}$ can be extended to $D_{j}$ in the following way: Let $p \in D_{j}$, for $n$ big enough, we have $F^{n}(p) \in W_{R, \rho, j}$. So we can define $\varphi(p)$ by $\varphi(p)=\varphi\left(F^{n}(p)-n\right.$ and the definition of $\varphi(p)$ does not depend on the choice of the integer $n$ such that $F^{n}(p) \in W_{R, \rho, j}$. 
Proposition 5.1. The Abel-Fatou's function $\varphi: D_{j} \rightarrow \mathbf{C}$ is surjective.

Proof: We have to show (if $R$ has been chosen big enough) that, for all $z \in \mathbf{C}$, there exists $n \in \mathbf{N}$ and $p \in W_{R, \rho, j}$ such that $\varphi(p)=z+n$. Using the definition of $\varphi$, and the notations in the proof of proposition (3.1), we have to show that there exists $(X, y) \in V_{R, \rho}$ such that

$$
u(X, y)=X-c \log X+u_{1}(x, y)=z+n .
$$

But for every fixed $y$ such that $\|y\|<\rho$, and for $n$ big enough, one can solve (5.1) in $z$ in $P_{R}$. This is a consequence of Rouché's theorem, for the equation (5.1) can be written

$$
X\left(1-\frac{1}{X}\left(c \log X+u_{1}(X, y)\right)\right)=z+n,
$$

and on the boundary $\operatorname{Re} X=R$, we have $\left|\frac{1}{X}\left(c \log X+u_{1}(x, y)\right)\right|<1$ (if $R$ is big enough). So the equation (5.1) has the same number of solutions as $X=z+n$.

We will now give a proof of the theorem 1.2 stated in the introduction.

Theorem 1.2. Let $F$ be a global automorphism in $\mathbf{C}^{2}$ with a fixed point $p$, such that $F_{(p)}^{\prime}$ has eigenvalues $\{1, \lambda\}$, with $|\lambda|<1$, and that $F-$ Id has a multiplicity $k+1$ in $p$. The attracting domain of $p$ has then $k$ components and each component is isomorphic to $\mathbf{C}^{2}$.

Proof: We first choose coordinates $(x, y)$ such that $F$ takes the form

$$
\left\{\begin{array}{l}
x_{1}=x\left(1-\frac{1}{k} x^{k}+a_{2 k} x^{2 k}+a_{2 k+1}(y) x^{2 k+1}+\ldots\right) \\
y_{1}=b y+b_{1} y x+\cdots+b_{k} y x^{k}+b_{k+1}(y) x^{k+1}+\ldots
\end{array} .\right.
$$

In fact, it is proved in $[\mathbf{U}]$ that for all integer $m$, coordinates $(x, y)$ can be chosen so that $y_{1}$ is expressed as

$$
y_{1}=b y+b_{1} y x+\cdots+b_{m} y x^{m}+b_{m+1}(y) x^{m+1}+\ldots,
$$

with $b_{j}$ constant for $0 \leq j \leq m$.

Let us fix $W=W_{R, \rho, j}$ a component of $D=D_{j}$ in a neighborhood of 0 chosen as section 3 in these coordinates. We want to prove that the open set

$$
D=\bigcup_{n=0}^{\infty} F^{-n}(W)
$$


is isomorphic to $\mathbf{C}^{2}$.

We first choose new coordinates in $W$. Let $\varphi$ be the Abel-Fatou function. We have seen in (4.3) that

$$
\varphi(x, y)=\frac{1}{x^{k}}-c \log x^{k}+v(x, y)
$$

with $v($,$) a holomorphic bounded function in W$. We define new coordinates in $W(s, y)$ where

$$
s=\frac{1}{x}\left(1-c x^{k} \log x^{k}+x^{k} v(x, y)\right)^{1 / k}=(\varphi(x, y))^{1 / k} .
$$

So

$$
s=\frac{1}{x}\left(1+O_{y}\left(\left|x^{k} \log x^{k}\right|\right)\right.
$$

and

$$
x=\frac{1}{s}+O_{y}\left(\frac{|\log s|}{|s|^{k+1}}\right) .
$$

In these coordinates $F$ takes the form

$$
\left\{\begin{array}{l}
s_{1}=s\left(1+\frac{1}{s^{k}}\right)^{1 / k}\left(\text { or } s_{1}^{k}=s^{k}+1\right) \\
y_{1}=b y+b_{1} y \frac{1}{s}+\cdots+b_{k} y \frac{1}{s^{k}}+O_{y}\left(\frac{|\log s|}{|s|^{k+1}}\right)
\end{array} .\right.
$$

We now follow the same method as Ueda. We will give a sketch of his proof adding the necessary modifications.

Let $D /\langle F\rangle$ be the quotient manifold of $D$ by the transformation group $\left\{F^{n}\right\}_{n \in Z}$ (this group acts discretely on $D$, because $F^{n}$ tends to $0 \notin D$ when $n$ goes to $+\infty)$.

Let $\pi: D \rightarrow D /\langle F\rangle$ be the projection and $E: \mathbf{C} \rightarrow \mathbf{C}^{*}$, the function $E(z)=\exp (2 i \pi z)$. Since the Abel-Fatou function satisfies

$$
\varphi(F(p))=\varphi(p)+1,
$$

one can define $\tilde{\varphi}: D /\langle F\rangle \rightarrow C^{*}$ such that the following diagram is commutative

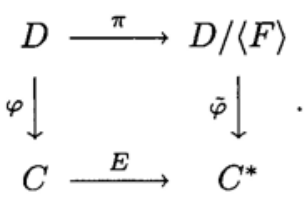


Define $B=\varphi(W)$. Let us consider the diagram obtained by restriction of the preceeding one to $\varphi^{-1}(B)$, that is

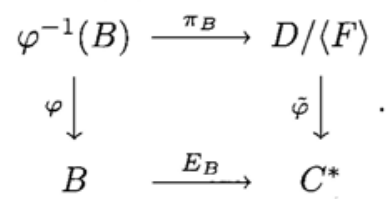

As follows from proposition (5.1), we know that $\mathbf{C}=\bigcup_{n=0}^{\infty}(B-n)$, so that the restriction

$$
E_{B}: B \rightarrow C^{*}
$$

is surjective. For $s \in B$, Ueda defines a holomorphic family of holomorphic functions $\psi_{s}: \varphi^{-1}(s) \rightarrow \mathbf{C}$ on the fibers of $\varphi$ which gives to $D /\langle F\rangle \rightarrow \mathbf{C}^{*}$ a fiber bundle structure with fibers isomorphic to $\mathbf{C}$ and with transition group the additive group of holomorphic functions on $\mathbf{C}^{*}$. This fiber bundle structure is necessarily trivial because $H^{1}\left(\mathbf{C}^{*}, O\right)=0$. Lifting this structure to $\varphi: D \rightarrow \mathbf{C}$ by $E$, we get a trivial fiber bundle structure on $D$ and this gives an isomorphism from $D$ to $\mathbf{C}^{2}$.

The definition of the $\psi_{s}$ 's is obtained by integrating on the fibers $s=$ Constant a holomorphic differential 1-form satisfying a functional equation, that we have now to define.

Definition of a holomorphic family of 1-forms on $W$.

We will define on $W$ a family of holomorphic differential 1-forms $\left\{\omega_{s}\right\}$ on the fiber of the Abel Fatou function depending holomorphically on $s$, of the form

$$
\omega_{s}(p)=\eta(s, y) d y=\eta(p) d y,
$$

with $\eta$ a holomorphic function in $B=\varphi(W)$ satisfying the functional equation

$$
\eta(F(p)) \frac{\partial y_{1}}{\partial y}(p)=\eta(p)
$$

We notice that

$$
\frac{\partial y_{n+1}}{\partial y}(p)=\frac{\partial y_{n}}{\partial y}(F(p)) \frac{\partial y_{1}}{\partial y}(p)
$$

So that if the sequence $\left\{\frac{\partial y_{n}}{\partial y}\right\}$ were uniformly converging, its limit would be a good candidate for $\eta$. But this is not the case since

$$
\begin{aligned}
\frac{\partial y_{n}}{\partial y} & =\frac{\partial y_{1}}{\partial y} \frac{\partial y_{2}}{\partial y_{1}} \cdots \frac{\partial y_{n}}{\partial y_{n-1}} \\
& =b^{n} \prod_{h=0}^{n-1}\left(1+\frac{b_{1}}{b} \frac{1}{s_{h}}+\cdots+\frac{b_{k}}{b} \frac{1}{s_{h}^{k}}+O_{y}\left(\frac{\left|\log s_{h}\right|}{\left|s_{h}\right|^{k+1}}\right)\right) .
\end{aligned}
$$


So we will replace the sequence $\left\{\frac{\partial y_{n}}{\partial y}\right\}$ by a sequence $\left\{h_{n} \frac{\partial y_{n}}{\partial y}\right\}$ where $\left\{h_{n}\right\}$ is a sequence of holomorphic functions such that

$$
h_{n}(F(p))=h_{n+1}(p),
$$

and $\left\{h_{n} \frac{\partial y_{n}}{\partial y}\right\}$ is uniformly convergent.

We can take $h_{n}(p)$ to be of the form

$$
h_{n}(p)=g(s) u(s) u\left(s_{1}\right) \ldots u\left(s_{n}\right)
$$

with $g$ and $u$ depending only on $s$, holomorphic in $W$ such that

$$
g(s) u(s)=g\left(s_{1}\right)
$$

and

$$
u(s)=b^{-1}\left(1+\frac{b_{1}}{b} \frac{1}{s}+\cdots+\frac{b_{k}}{b} \frac{1}{s^{k}}\right)^{-1}+O\left(\frac{1}{|s|^{k+1}}\right)
$$

The condition (5.11) implies indeed (5.10) and the condition (5.12) implies that

$$
h_{n}(p) \frac{\partial y_{n}}{\partial y}=\prod_{h=0}^{n-1}\left(1+O_{y}\left(\frac{\left|\log s_{h}\right|}{\left|s_{h}\right|^{k+1}}\right)\right)=\prod_{h=0}^{n-1}\left(1+O\left(\frac{\log h}{h^{1+1 / k}}\right)\right) .
$$

So that $\eta$ will be defined by a uniformly convergent infinite product.

We have then to show that there exists a function $g$ holomorphic in $B$ such that $u(s)=g\left(s_{1}\right) / g(s)$ satisfies (5.12). We define $g$ as a product of three functions

$$
g=g_{1} \cdot g_{2} \cdot g_{3}
$$

where $g_{1}(s)=b^{-s^{k}}$ (for any choice of $\log b$ ). In fact, this gives

$$
\frac{g_{1}\left(s_{1}\right)}{g_{1}(s)}=b^{-1}
$$

We then choose a function $g_{2}$ satisfying

$$
\frac{g_{2}\left(s_{1}\right)}{g_{2}(s)}=\left(1+\frac{b_{1}}{b} \frac{1}{s}+\cdots+\frac{b_{k-1}}{b} \frac{1}{s^{k-1}}\right)^{-1}+O\left(\frac{1}{s^{k}}\right) .
$$

The existence of $g_{2}$ is proved by the following lemma 
Lemma 5.3. For any $\left(c_{1}, c_{2}, \ldots, c_{k-1}\right) \in \mathbf{C}^{k-1}$, there exists a polynomial in $\frac{1}{s}$

$$
P\left(\frac{1}{s}\right)=\frac{a_{1}}{s}+\frac{a_{2}}{s^{2}}+\cdots+\frac{a_{k-1}}{s^{k-1}}
$$

such that $h(s)=\exp \left(s^{k} P\left(\frac{1}{s}\right)\right)$ satisfies

$$
\frac{h\left(s_{1}\right)}{h(s)}=1+\frac{c_{1}}{s}+\frac{c_{2}}{s^{2}}+\cdots+\frac{c_{k-1}}{s^{k-1}}+O\left(\frac{1}{s^{k}}\right) .
$$

Proof: From

$$
s_{1}^{k}=s^{k}+1,
$$

we get

$$
\frac{1}{s_{1}}=\frac{1}{s}-\frac{1}{k} \frac{1}{s^{k+1}}+O\left(\frac{1}{s^{2 k+1}}\right)
$$

so for a polynomial $P$

$$
P\left(\frac{1}{s_{1}}\right)=P\left(\frac{1}{s}\right)-\frac{1}{k} \frac{1}{s^{k+1}} P^{\prime}\left(\frac{1}{s}\right)+O\left(\frac{1}{s^{2 k+1}}\right) .
$$

From that we deduce

$$
\begin{aligned}
s_{1}^{k} P\left(\frac{1}{s_{1}}\right)=s^{k} P\left(\frac{1}{s}\right) & =s^{k}\left(P\left(\frac{1}{s_{1}}\right)-P\left(\frac{1}{s}\right)\right)+P\left(\frac{1}{s_{1}}\right) \\
& =P\left(\frac{1}{s}\right)-\frac{1}{k s} P^{\prime}\left(\frac{1}{s}\right)+O\left(\frac{1}{s^{k+1}}\right) \\
& =\sum_{j=1}^{k-1}\left(1-\frac{j}{k}\right) a_{j} \frac{1}{s^{j}}+O\left(\frac{1}{s^{k+1}}\right) .
\end{aligned}
$$

We can then compute the $a_{j}$ 's by identifying the expression above with the polynomial part of degree $\leq k-1$ in the Taylor expansion of

$$
-\log \left(1+\frac{c_{1}}{s}+\frac{c_{2}}{s^{2}}+\cdots+\frac{c_{k-1}}{s^{k-1}}\right)
$$

at infinity.

Choice of $g_{3}$. It is a consequence of the choice of $g_{1}$ and $g_{2}$ that it exists $c$ such that

$$
\frac{g_{1}\left(s_{1}\right)}{g_{1}(s)} \frac{g_{2}\left(s_{1}\right)}{g_{2}(s)} b\left(1+\frac{b_{1}}{b} \frac{1}{s}+\cdots+\frac{b_{k}}{b} \frac{1}{s^{k}}\right)=1+\frac{c}{s^{k}}+O\left(\frac{1}{s^{k+1}}\right) .
$$


So we need a function $g_{3}$ such that

$$
\frac{g_{3}\left(s_{1}\right)}{g_{3}(s)}=1-\frac{c}{s^{k}}+O\left(\frac{1}{s^{k+1}}\right) .
$$

We can take $g_{3}$ defined by $g_{3}(s)=s^{-k c}=\exp \left(-c \log s^{k}\right)$ (with the branch of logarithm which is real on positive numbers). Indeed, we get

$$
\frac{g_{3}\left(s_{1}\right)}{g_{3}(s)}=\left(1+\frac{1}{s^{k}}\right)^{-c}=1-\frac{c}{s^{k}}+O\left(\frac{1}{s^{k+1}}\right) .
$$

Once $\omega_{s}$ is defined in $W$, we just follow the construction of Ueda to define the $\psi_{s}$. We recall this construction for reader's convenience.

Construction of $\psi_{s}: \varphi^{-1}(s) \rightarrow \mathbf{C}$.

We have seen that if $p \in D$, for $n$ big enough, $s=F^{n}(p)$ is in $B$ and the fiber $D \cap \varphi^{-1}(s)$ contains a disk $\Delta_{\rho}=\{y \in \mathbf{C} ;\|y\|<\rho\}$. So we can fist suppose that $W$ is a set of the form $W=B \times \Delta_{\rho}$ and that we still have $D=\bigcup_{n=0}^{\infty} F^{-n}(W)$. We first define $\psi_{s}(p)$ when $p$ is in $W$ and $\varphi(p)=s$ by integrating the form $\eta(p) d y$ on the fiber $s=$ constant, along a path joining $p_{0}=(s, 0)$ to $p=(s, y(p))$ in $\{s\} \times \Delta_{\rho}$

$$
\psi_{s}(p)=\int_{0}^{y(p)} \eta(s, y) d y
$$

From the functional equation (5.8) verified by $\eta$ we get

$$
\begin{aligned}
\psi_{s_{1}}(F(p)) & \left.=\int_{0}^{y(F(p))} \eta\left(s_{1}, y_{1}\right)\right) d y_{1} \\
& =\int_{y\left(F\left(p_{0}\right)\right)}^{y(F(p))} \eta\left(s_{1}, y_{1}\right) d y_{1}+\int_{0}^{y\left(F\left(p_{0}\right)\right)} \eta\left(s_{1}, y\right) d y \\
& =\psi_{s}(p)+h(s)
\end{aligned}
$$

where $h(s)=\int_{0}^{y\left(F\left(p_{0}\right)\right)} \eta\left(s_{1}, y\right) d y$ is a holomorphic function of $s$ in $B$.

We get in this way a holomorphic function $\psi: W \rightarrow C$ defined by $\psi(p)=\psi_{s}(p)$ for $s=(\varphi(p))^{1 / k}$ such that

$$
\psi(p)=\psi_{s}(p)=\psi_{s_{1}}(f(p))-h(s) .
$$

The relation (5.15) allows the extension of the definition of $\psi$ to $\varphi^{-1}(B)$ in the following way: let $p \in \varphi^{-1}(B)$. For $n$ big enough, we have $F^{n}(p) \in$ $W$ and we define $\psi(p)$ by the formula

$$
\psi(p)=\psi\left(F^{n}(p)\right)-\left(h(s)+h\left(s_{1}\right)+\cdots+h\left(s_{n}\right)\right)
$$

where $s_{j}=\left(\varphi\left(f^{j}(p)\right)\right)^{1 / k}$ for $j=0,1, \ldots, n$. It is clear that the definition doesn't depend on $n$ and that the function $\psi$ is holomorphic in $\varphi^{-1}(B)$. 
Proposition 5.4 (Ueda). For all $s \in B, \psi_{s}: \varphi^{-1}(s) \rightarrow \mathbf{C}$ is an isomorphism.

Proof: Let us prove first the injectivity of $\psi_{s}$ : Let $p$ and $p^{\prime} \in \varphi^{-1}(s)$ such that $\psi_{s}(p)=\psi_{s}\left(p^{\prime}\right)$. According to property (5.16), one can assume that $p$ and $p^{\prime}$ are in $W$ and that we have with $s$ big and $y(p)$ and $y\left(p^{\prime}\right)$ small

$$
\int_{0}^{y(p)} \eta(s, y) d y=\int_{0}^{y\left(p^{\prime}\right)} \eta(s, y) d y .
$$

The results is just a consequence of the fact that the function $\int_{0}^{y(p)} \eta(s, y) d y$ is holomorphic in $y(p)$ and has a derivative $\eta(s, 0) \neq 0$.

To prove the surjectivity, we remark that $\eta(s, 0)_{\sim} b^{-s k} s^{-k c}$ when $s \rightarrow$ $\infty$ and as

$$
h(s)=\int_{0}^{y\left(F\left(p_{0}\right)\right)} \eta\left(s_{1}, y\right) d y
$$

with $y\left(F\left(p_{0}\right)\right)=y_{1}(s, 0)=\frac{b_{k+1}(0)}{s^{k+1}}+\ldots$ (see the form of $F$ in coordinates $(s, y))$. We have

$$
h(s)_{\sim} \eta(s, 0) \frac{b_{k+1}(0)}{s^{k+1}} .
$$

We deduce from this that the image $\psi_{s}(W)$ contains a disk centered at 0 with radius $\varepsilon \rho\left|b^{-s^{k}} s^{-k c}\right|$ for some constant $\varepsilon>0$. The relation (5.16) then implies that the image of $F^{-n}(W) \cap \varphi^{-1}(s)$ contains a disk centered in $\zeta_{n}=h(s)+h\left(s_{1}\right)+\cdots+h\left(s_{n}\right)$ and with radius $R_{n}=\varepsilon \rho\left|b^{-s_{n^{k}}} s_{n^{-k c}}\right|$. An elementary calculation proves that $R_{n}$ et $\left|\zeta_{n}\right|$ tend to $+\infty$ while $\frac{\zeta_{n}}{R_{n}} \rightarrow 0$, so the union of the disks $D\left(\zeta_{n}, R_{n}\right)$ contained in the image of $\varphi^{-1}(s)$ is equal to $\mathbf{C}$.

It is then easy using the $\psi_{s}$ 's to define a structure of locally trivial fiber bundle on $D /\langle F\rangle \rightarrow \mathbf{C}^{*}$, with fibers isomorphic to $\mathbf{C}$ and with structure group, the group of translations by holomorphic functions in $s$. This ends the proof of the existence of an isomorphism from $D$ to $\mathbf{C}^{2}$.

\section{References}

[B] Beardon A. F., "Iteration of rational functions," Springer, New York, Berlin and Heidelberg, 1991.

[C] Chirka E. M., "Complex Analytic sets," vol. 46, Kluwer Academic Publishers, Dadrech, Boston, London, 1989. Translation in English. 
[F] FATOU P., Substitutions analytiques et equations fonctionnelles à deux variables, An. Sc. Ec. N. (1924), 67-142.

[R] Ruelle D., "Elements of differentiable dynamics and bifurcation theory," Academic Press, Boston, 1989.

[U] UEDA T., Local structure of analytic transformations of two complex variables I, J. Math. Kyoto Univ. 26(2) (1986), 233-261.

\author{
Département de Mathématiques \\ Bât. 425 \\ Unité Associée CNRS \\ Université de Paris-Sud \\ 91405 Orsay Cedex \\ FRANCE
}

Rebut el 5 d'Octubre de 1994 